ORIGINAL ARTICLE

\title{
Childhood constipation is not associated with characteristic fingerprint patterns
}

\author{
C R Jackson, B Anderson, B Jaffray
}

Arch Dis Child 2003;88:1076-1077

See end of article for authors' affiliations

......................

Correspondence to: Mr B Jaffray, Senior Lecturer in Paediatric Surgery, School of Clinical Medical Sciences (Child Health), Sir James Spence Institute, University of Newcastle upon Tyne, Newcastle upon Tyne NEI 4LP, UK :

bruce.jaffray@ncl.ac.uk

Accepted 8 October 2003
Background: It has been suggested that there is an association between simple arch fingerprint patterns and severe childhood constipation. If real, this association might be useful to predict which children have a poor prognosis.

Aim: To see how many severely constipated children have simple arches, compared to non-constipated controls and their first degree relatives.

Methods: Fingerprints were classified by two blinded assessors in 30 children requiring surgery for refractory constipation, and 30 children with appendicitis, and the first degree relatives of both groups. Colonic transit times and clinical outcomes were also evaluated among constipated children.

Results: At least one simple arch was found in similar numbers of constipated children (13\%) and their families (16\%), and control children (7\%) and their families (13\%). Arch positivity was commoner among relatives of arch positive (6/6) than arch negative children (14/54), regardless of bowel history. Arch positivity did not identify children with prolonged transit times, nor those who required colectomy.

Conclusions: Fingerprint patterns are not associated with severe childhood constipation, do not aid their management, and do not support a genetic aetiology for this problem.
C onstipation is common in children, ${ }^{1}$ and though most cases respond to dietary manipulation and laxatives, some have constipation and soiling into adulthood. ${ }^{2}$ It would be valuable to predict these non-responders early.

Fingerprint patterns are genetically determined and conditions such as trisomy 18 or 21 are associated with unusual dermatoglyphics. ${ }^{3}{ }^{4}$ Two studies have linked early onset constipation with an abnormal frequency of simple arches, ${ }^{56}$ with early onset being described as under 10 years old and within the first few months of life respectively. Further work has suggested an association with chronic intestinal pseudoobstruction. ${ }^{78}$ The implication is that some constipated patients may have a genetic basis for their condition. Others have been unable to confirm the association. ${ }^{9}$ If there is a genetic aetiology to some cases of constipation, patients are likely to present in childhood and to have severe symptoms, such as the ones our unit treats with antegrade continent enema surgery (ACE) after the failure of several years medical management. ${ }^{10}$ We speculated that if there are cases of congenital constipation, they are likely to be found in such a cohort. We therefore compared the fingerprints of these children to those of a control group of children with appendicitis, which has no known genetic basis.

\section{PATIENTS AND METHODS}

Thirty consecutive children (13 boys) who had severe chronic constipation and a ganglionic rectal biopsy, requiring formation of an ACE were recruited. In all cases constipation was labelled as idiopathic, having excluded organic causes such as Hirschsprung's disease. There were no major coexisting diseases, including chromosomal disorders, in any child. The mean age at onset of symptoms was 2.65 years (range 3 months to 8 years). Their mean age at presentation to a paediatrician for management of constipation was 4 years and the mean age at surgery was 10 years. All had received several years of laxative therapy, most had had in-patient rectal disimpaction and enemata, several had required nasogastric administration of bowel preparation fluids, and involvement of a clinical psychologist was common. Heavy daily soiling was usual. The controls were 30 consecutive children (19 boys) undergoing appendectomy for histologically confirmed appendicitis. Their case notes were reviewed to ascertain whether they had been treated for constipation, and the case notes of both groups were reviewed for evidence of a family history of constipation. First degree relatives of both groups also had dermatoglyphic studies.

Fingerprints were recorded using an inkless technique ${ }^{3}$ using standard equipment (Printkits, K9 Scene of Crime Equipment Ltd, Northampton, UK) by a dedicated nurse (BA). Two individuals (CRJ, BJ) each assessed the records twice, blinded to the patient category, and assigned the patterns as loop or tented arch, whorl, simple arch, or as unclassifiable. Subjects with at least one digit with a simple arch was categorised as "arch positive".

Among the ACE group, total colonic transit times were measured using standard methodology before surgery, ${ }^{11}$ and their success in adapting to colonic lavage was recorded.

\section{Statistical analysis}

Previous studies have suggested that arch positivity might be seen in $53 \%$ of constipated patients and $11 \%$ of controls. ${ }^{5}$ For $\alpha($ two sided $)=0.05$ and $\beta=0.1$, a sample size of 30 is required. ${ }^{12}$ Statistical comparison of proportions utilised the $\chi^{2}$ test. Comparison of medians used the Mann-Whitney test. The intra-observer and inter-observer reproducibility of the assignation of dermatoglyphic patterns was assessed by the kappa statistic, ${ }^{13}$ using SSPS statistical software.

The hospital's ethical committee approved the protocol, and written informed consent was obtained from parents.

\section{RESULTS}

We recorded the fingerprints of 229 individuals. The 30 constipated children had 87 first degree relatives; and the 30 post-appendectomy children had 82. Among the children with constipation, eight had a positive family history, of whom three were arch positive. No control children or their families had a history of constipation.

Most digits had loops or tented arches (71.4\%), or whorls (18.1\%); $5.5 \%$ could not be classified because of poor print quality, and only $4.5 \%$ had simple arches, most commonly on the index fingers. The $\kappa$ value for inter-assessor reproducibility of dermatoglyphic assignment was 0.70 , and for intraassessor reproducibility was 0.89 . 
Among the children studied, 4/30 (13\%) of the constipated group had one or more simple arches, compared with $2 / 30$ $(7 \%)$ of the controls $\left(\chi^{2}=0.74, p=0.4\right)$. Similar frequencies were seen among the first degree relatives, with 14/87 (16\%) of the families of constipated children having simple arches, and $11 / 82(13 \%)$ of the control families $\left(\chi^{2}=0.24, p=0.6\right)$. All the families of the six arch positive children also had members with simple arches, whether the children were constipated or not, while only 14 families of the 54 arch negative children had members with simple arches $\left(\chi^{2}=13.3, p<0.001\right)$.

Among constipated children, the median colonic transit times were 102 hours (range 70-134) in arch positive constipated cases, and 77 hours (range 42-144) in arch negative cases $(p=0.7){ }^{1}$

Four of the constipated children have gone on to colectomy for intractable constipation, having failed to establish a satisfactory pattern of defecation with colonic lavage. Review of the colectomy specimens showed melanosis coli in one specimen, but there were no other pathological features. The presence of simple arches failed to predict this subset of children (one arch positive, three arch negative).

\section{DISCUSSION}

Simple arches are inherited dominantly with incomplete penetrance, ${ }^{14}$ and unusual dermatoglyphics are a feature of some chromosomal disorders, such as Turner's and Down's syndrome. ${ }^{15}$ Previous research into the association between simple arches and constipation has revealed conflicting results. ${ }^{569}$ The current study suggests that there is no such association.

If some constipated patients suffer from a genetic disorder, it would seem reasonable to assume that their symptoms would begin in childhood. However, the majority of constipated children suffer from a mild, self-limiting condition, with a high chance of cure. ${ }^{16}$ Our subset of children are particularly likely to reveal a genetic predisposition if it exists because they were selected for surgery for early onset severe, resistant symptoms.

In the study of Drongowski and Coran, ${ }^{9}$ which failed to find an association between simple arches and constipation, the control group showed a $29.8 \%$ incidence of simple arches, when population based studies suggest that the expected incidence of simple arches should be much lower. ${ }^{17}$ The aberrant nature of this control group would lessen their chance of detecting an association between constipation and dermatoglyphic patterns. In the current study, by contrast, the incidence of the three patterns of loop, whorl, and arch are in accordance with studies of the British population. ${ }^{17}$ For this reason we were disinclined to accept the conclusion of Drongowski and Coran as a definitive answer to the question of an association between constipation and simple arches.

In the original report of association between simple arches and early onset constipation (before age 10), simple arches were found in $53 \%$ of patients with early onset constipation, compared with $13 \%$ of patients with later onset constipation and $11 \%$ of non-constipated patients attending a clinic for gastrointestinal consultation..$^{5}$ The incidence of simple arches in the current study is lower than the incidence seen in early onset constipated patients in Gottleib and Schuster's study, and is similar to their control group. The methodology of Gottleib and Schuster's study may be criticised, since they studied the fingerprints of 155 patients attending a gastrointestinal clinic, and then analysed the symptoms of patients according to their arch status. Clearly, they were selecting arch positive patients, and in the context of patients attending a gastrointestinal clinic there is a high chance that such patients will volunteer symptoms of constipation. We believe that this methodological flaw explains their findings. The current study adopts a more robust methodology of selecting severely constipated children, ascertaining their arch status, and comparing them with an appropriate group of non-constipated children.
The other study purporting to show an association between chronic constipation and simple arches fails to give details on how constipated children were selected for study, and it is therefore difficult to explain their finding of $38 \%$ of such children showing simple arches. ${ }^{6}$ Our own experience with assigning dermatoglyphic patterns makes us aware of the difficulty in distinguishing simple arches from the more common tented arch, and we would speculate that poor accuracy of this test might be a source of confusion. However, the precision of dermatoglyphic assessment is high, with $\kappa$ values of 0.7 and 0.89 for inter- and intra-observer reproducibility respectively.

In addition to finding that dermatoglyphic analysis shed no light on the aetiology of constipation in children, we also found it of no practical use in their management. Arch positivity identifies neither those with the most severe constipation, nor those who fail to adapt to colonic lavage. However, we recognise that the very small number of children in the arch positive group who had undergone measurement of colonic transit time, or progressed to colectomy means we cannot be certain about this.

This study does not prove that some cases of severe childhood constipation do not have a genetic component, which is indeed supported by other lines of research, ${ }^{18}$ and the significant family history seen in the constipated group in this study and others ${ }^{19}$ may be evidence for a genetic component. However, if such a condition does exist, it is not associated with characteristic dermatoglyphics.

\section{ACKNOWLEDGEMENTS}

We are grateful to Dr E Eastham and Dr M Coulthard for helpful suggestions on study design and manuscript presentation. BA was funded by a grant from the Newcastle Hospital's Special Trustees. This work was presented to the Association of Surgeons of Great Britain and Ireland meeting in Manchester, 2003.

\section{Authors' affiliations \\ C R Jackson, B Anderson, B Jaffray, University of Newcastle upon Tyne,} UK

\section{REFERENCES}

1 Loening-Baucke V. Chronic constipation in children. Gastroenterology 1993; 105: 1557-64.

2 van Ginkel R, Reitsma JB, Buller HA, et al. Childhood constipation:

longitudinal follow-up beyond puberty. Gastroenterology 2003;125:357-63.

3 Gibbs RC. Fundamentals of dermatoglyphics. Arch Dermatol 1967;96:721-5.

4 Verbov J. Clinical significance and genetics of epidermal ridges - a review of dermatoglyphics. J Invest Dermatol 1970;54:261-71.

5 Gottlieb SH, Schuster MM. Dermatoglyphic (fingerprint) evidence for a congenital syndrome of early onset constipation and abdominal pain. Gastroenterology 1986;91:428-32.

6 Staiano A, Andreotti MR, Perrotta V, et al. Prevalence of digital arches in children with abdominal pain and constipation. J Pediatr 1990;1 17:435-6.

7 Pulliam TJ, Schuster MM. Congenital markers for chronic intestinal pseudoobstruction. Am J Gastroenterol 1995;90:922-6.

8 Chakravarti A, Blanton S, Kendall BJ, et al. Cosegregation of familial intestinal pseudoobstruction and presence of digital arches in a large multigenerationa pedigree. Dig Dis Sci 1996;41:1429-33.

9 Drongowski RA, Coran AG. Dermatoglyphic patterns in children with chronic constipation. Dig Dis Sci 1995;40:1420-2.

10 Marshall J, Hutson JM, Anticich N, et al. Antegrade continence enemas in the treatment of slow-transit constipation. J Pediatr Surg 2001;36:1227-30.

11 Metcalf AM, Phillips SF, Zinmeister AR, et al. Simplified assessment of segmental colonic transit. Gastroenterology 1987:92:40-7.

12 Hulley SB, Cummings SR, Browner WS. Designing clinical research: an epidemiologic approach, 2nd edn. Philadelphia: Lippincott Williams \& Wilkins 2001

13 Cohen J. Coefficient of agreement for nominal scales. Electroencephalogr Clin Neurophysiol 1960;20:37-46.

14 Anderson MW, Bonne-Tamir B, Carmelli D, et al. Linkage analysis and the inheritance of arches in a Habbanite isolate. Am J Hum Genet 1979;31:620-9.

15 Penrose LS. Fingerprints, palms and chromosomes. Nature 1963;197:933-8.

6 Loening-Baucke V. Constipation in early childhood: patient characteristics, treatment, and longterm follow up. Gut 1993;34:1400-4.

17 Holt SB. Quantitative genetics of finger-print patterns. Br Med Bull $1961 ; 17: 247$.

18 Knowles $\mathrm{CH}$, Scott S, Wellmer A, et al. Sensory and autonomic neuropathy in patients with idiopathic slow-transit constipation. Br J Surg 1999:86:54-60.

19 Staiano A, Andreotti MR, Greco L, et al. Long-term follow-up of children with chronic idiopathic constipation. Dig Dis Sci 1994;39:561-4. 